\title{
A REVIEW OF MULTIHOP BASED MEDIUM ACCESS CONTROL (MAC) PROTOCOL TO TACKLE BOTH MULTIPLE ACCESS AND MULTIHOP ISSUES IN WIRELESS MESH NETWORK (WMN)
}

\author{
Vigneswara Rao Gannapathy ${ }^{1}$, Lim Kim Chuan ${ }^{2}$, Siva Kumar Subramaniam³, \\ Zahriladha Bin Zakaria ${ }^{4}$ \\ ${ }^{I}$ Senior Lecturer, Department of Electronics and Computer Engineering, Universiti Teknikal Malaysia Melaka, \\ Melaka (UTeM), Malaysia \\ ${ }^{3}$ Senior Lecturer, Department of Electronics and Computer Engineering, Universiti Teknikal Malaysia Melaka, \\ Melaka (UTeM), Malaysia \\ ${ }^{3}$ Senior Lecturer, Department of Electronics and Computer Engineering, Universiti Teknikal Malaysia Melaka, \\ Melaka (UTeM), Malaysia \\ ${ }^{4}$ Associate Professor, Department of Electronics and Computer Engineering, Universiti Teknikal Malaysia Melaka, \\ Melaka (UTeM), Malaysia
}

\begin{abstract}
Multihop WMN plays an important role in the next-generation wireless communication. It promised a solution to provide ubiquitous wireless access at low cost and with architecture that easy to be deployed and maintained. A big challenge in designing WMN is to utilize the shared medium, the wireless communication channel, effectively. MAC protocol, the shared medium access controller, therefore plays a critical role in the channel utilization. The better the channel is utilized in WMN, the better is the performance of the WMN. One of the techniques to improve channel utilization is by enabling the concurrent transmission and providing an efficient forwarding operation. However, the existing IEEE 802.11 MAC does not supports those operations in WMN thus degrades the throughput significantly. To date, various multihop based MAC protocols was developed and proposed by researchers with the aim to regulate and solve access issues among all radio nodes in the network. This article presents an exhaustive survey of multihop based contention MAC protocols that proposed to solve exposed node and forwarding issues in multihop WMN. Besides that, the comparison, their operations, advantages and disadvantages of all identified MAC protocols also will be explained and presented in this article.
\end{abstract}

Keywords: Multihop, Wireless Mesh Network (WMN), Medium Access Control (MAC), Contention Based Protocol, Network Allocation Vector (NAV)

\section{INTRODUCTION}

The establishment of access rights proposed in IEEE 802.11 MAC [1] is far more difficult in multihop WMN. It is observed by many researchers that the throughput of the IEEE 802.11 MAC protocol drops significantly when applied directly in multihop networks such as WMN [2, 3 \& 4]. It's well noted that the existing MAC protocol that initially proposed for Wireless Local Area Network (WLAN) suffered from exposed node and forwarding issues when implemented in multihop wireless network. The IEEE 802.11 MAC protocol, tackle the issues of hidden node problem by employing a four way handshake of RTS-CTSDATA-ACK frames.

According to the handshake mechanism, the source node will send the RTS and DATA frame while the destination node will respond with CTS and ACK frames. All the nodes which are in the range either source or the destination nodes have to remain silent and refrain from transmit any frame until entire transmission is completed. This virtual carrier sensing mechanism is to avoid the collision of DATA frame at recipient node and CTS or ACK frames at sender node. Even though this mechanism alleviates the hidden node problem yet the exposed node problem still remain unsolved.

The IEEE 802.11 MAC protocol effectively precludes other neighboring nodes from involving in transmission activity during the entire duration of ongoing frame transmission. This will introduced the problem called exposed node thus degrades the performance accordingly. These nodes will remain silent and refrain from initiate the transmission for the duration as specified in their NAV. In addition, when the number of competing nodes increases in the network, thus the number of exposed node also increases accordingly.

The exposed node problem can be elaborated more by referring to Figure 1. After proper exchanged of RTS/CTS control frames between node R1 and A, they will begins their DATA transmission. During this entire frame 
transmission, node $\mathrm{B}$ does not allow to receive the frame from any of its neighboring nodes since it already silenced by CTS control frame from node A (i.e. virtual carrier sensing Mechanism). This is known as exposed node problem. By right, node $\mathrm{B}$ can be allowed to receive the DATA frame from R2 since it doesn't conflict with the ongoing transmission from node R1 to node A.

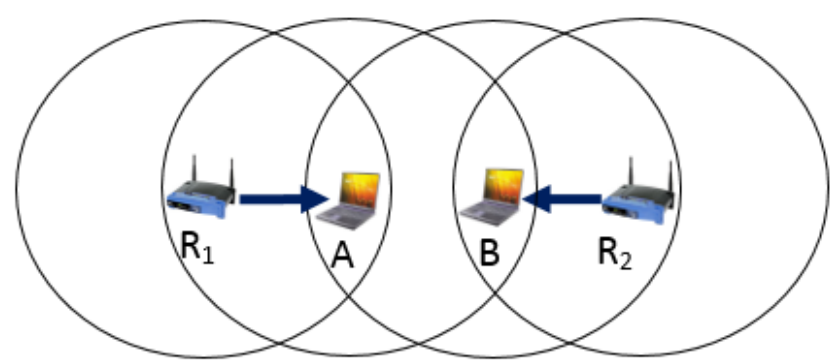

Fig. 1: Exposed Node Problem Cause by Virtual Carrier Sensing (Scenario A)

Figure 2 shows different scenario of exposed node problem. After proper exchanged of RTS/CTS control frames between node $\mathrm{A}$ and R1, they will begins their DATA transmission accordingly. During this entire frame transmission, node $\mathrm{B}$ does not allow to involve in transmission activity with any of its neighboring nodes since it already silenced by RTS control frame from node A (i.e. virtual carrier sensing Mechanism). This is also known as exposed node problem. By right, node $\mathrm{B}$ can be allowed to transmit the DATA frame to R2 concurrently since it doesn't conflict with the ongoing transmission from node A to node $\mathrm{R} 1$.

Thus, under the IEEE 802.11 DCF MAC protocol, it effectively precludes other one hop neighboring nodes from involve in transmission activity during the entire duration of ongoing frame transmission and cause exposed node problem thus degrades the network performance.

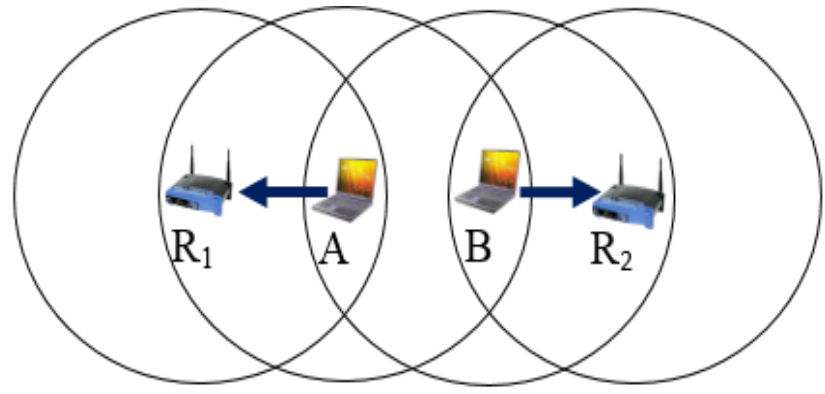

Fig 2: Exposed Node Problem Cause by Virtual Carrier Sensing (Scenario B)

Moreover, in multihop WMN communication, if a node needs to communicate with another node (i.e. mesh router or internet gateway) that is not within its range, then it will relays the frame to its adjacent mesh routers. The frame is then will be relayed over multiple hops until it reaches an internet gateway. Then the internet gateway will forward the frame to destination node. The current IEEE 802.11 MAC protocol is only designed for single hop communication (i.e. transmit and receive the frame with the nodes directly associate with it). This protocol does not support the forwarding operating (i.e. receive a frame and immediately forward it to next relaying node) [5]. Thus due to this reason, the current protocol is not suit when implemented in multihop WMN communication.

According to IEEE 802.11 MAC protocol, to relay a frame over multihop, a channel access sequence (i.e. RTS-CTSDATA-ACK) is required at each hop. By referring to the example in Figure 3, node A has frame to transmit to node $\mathrm{R} 5$ via intermediate nodes $\mathrm{R} 1, \mathrm{R} 2, \mathrm{R} 3$ and $\mathrm{R} 4$ which is serve as relaying nodes. Since node A relays its frame over multiple hops until it reached its destination, a signaling overhead introduced at each intermediate hop degrade the performance of WMN accordingly. The signaling overhead will increase proportionally with the increase of hops. As IEEE 802.11 MAC was developed for one-hop communication, later the researchers are observed that this protocol performs poorly in multihop communication. Figure 3 shows the forwarding operation when the frame transmitted in multihop environment.

The exposed node problem and inefficient forwarding operation result in unnecessary reduction in channel utilization and larger signaling overhead respectively. In multihop environment, the performance would degrade dramatically due to these problems. A good MAC protocol for multihop WMN should design to efficiently share the channel and boost the performance.

Therefore, to suit and support the multihop WMN, several MAC protocols have been developed by the researchers. The main aim of the developed protocols is to improve the overall throughput of a multihop WMN. The following section will discuss some related works that had been proposed by researchers in order to solve the exposed node problem by enabling the concurrent transmission. In addition, the following section also will discuss some of works that had been proposed by researchers in order to provide an efficient forwarding operation. The efficient forwarding operation is expected to reduce the signaling overhead and boosts the network throughput.

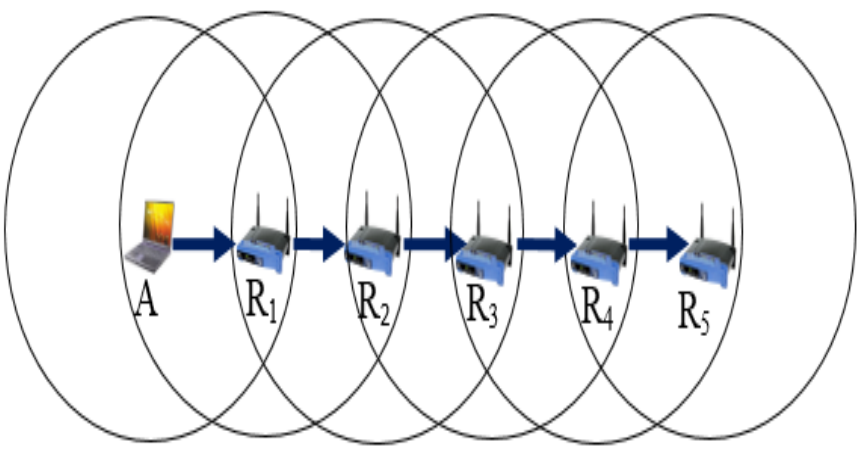

Fig 3: Forwarding Operation in Multihop Environment 


\section{CONCURRENT TRANSMISSION ACCESS PROTOCOLS}

The concurrent transmission approaches have been proposed by many researchers as a transmission strategy to combat exposed node problems which is common in IEEE 802.11 MAC protocol. Its restrictive nature to disallow other neighboring nodes from involves in the transmission activity until the ongoing transmission complete degrade the throughput of WMN significantly. The throughput can be improved by using the concurrent transmission approaches. Thus, it can achieve better channel utilization and spatial reuse. By enabling the concurrency, it boosts its throughput and also alleviates the exposed node problem. The following section will discuss some related approaches that had been proposed to solve exposed node problem by enabling concurrent transmission approach thus gain better channel utilization.

\subsection{Opportunistic Approach in Mitigating Exposed}

\section{Node Problem}

One interesting work has proposed in [6] to mitigate the expose node problem. The nodes implemented with this protocol will recognize themselves as an exposed node by hearing the basic sequence of frames (i.e. the RTS control frame followed by the DATA frame from the same node) and opportunistically schedule concurrent transmission whenever possible. For example, when the exposed nodes (i.e. E1, E2 and E3) in the Figure 4 overhear the basic sequence of frames, it will align their DATA frame transmission with ongoing transmission. This communication does not require any additional RTS/CTS exchange.

All exposed nodes (known as secondary transmission in this work) will tries to squeeze its transmission with primary node when it's DATA transmission is in progress. This approach effectively preclude from schedule secondary transmission, when multiple transmissions are in progress.

However the protocol enables the concurrency by the exposed node which is caused by the NAV RTS only. A part from that, the frame size of the primary transmission must be larger than the frame size of secondary transmission. This is another constraint that was highlighted by the author in this work to enable the concurrent transmission successfully. Since the frame size of primary transmission is larger than the secondary transmission, thus the secondary transmission is required to defer for some amount of time before finishing its transmission. This to make sure both primary and secondary transmission can finish exactly at the same time before commencing the ACK transmission. This deferral is to avoid the collision between DATA and ACK frames.

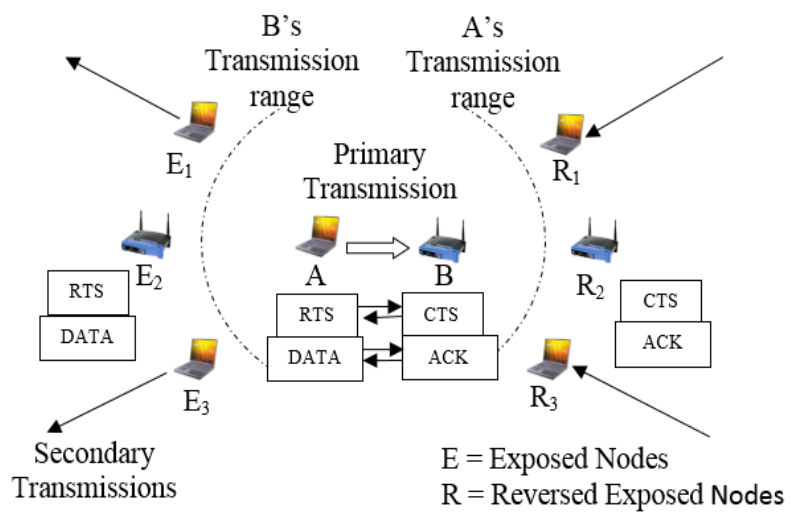

Fig 4: Opportunistic Approach in Mitigating Exposed Node Problem

\subsection{Multiple Access Collision Avoidance with Parallel Transmission (MACA-P)}

The earliest work on enabling concurrent transmission in WMN is Cooperative Medium Access Scheme [7] that contributes to significant improvement in the overall throughput of WMN. This protocol is improved version of [5], with the introduction of Multiple Access Collision Avoidance-Parallel or MACA-P protocol. Figure 5 shows the pattern of the concurrent transmission that could be allowed in MACA-P without the collision.

This mechanism improves the utilization of channel by adopting spatial-reuse technique. A 'control gap' is introduced between the exchange of RTS/CTS control frames and subsequent exchange of DATA/ACK frames. The control gap will begin at first sender - receiver pair of communicating node (i.e. master node). All other neighboring nodes will be exploited by RTS from master node to complete their own RTS/CTS within this control gap and to align their DATA/ACK transmission with the DATA and ACK phases of master node. In other word, this control gap is introduced to synchronize the DATA and ACK period of all concurrent transmission to avoid them colliding between each other (i.e. DATA frame of a transmission and ACK frame of another transmission). To achieve this DATA and ACK alignment, the RTS and CTS control frames were designed to carry the start time of DATA and ACK transmission. The detail refinements of MACA-P are described in [7 \& 8].

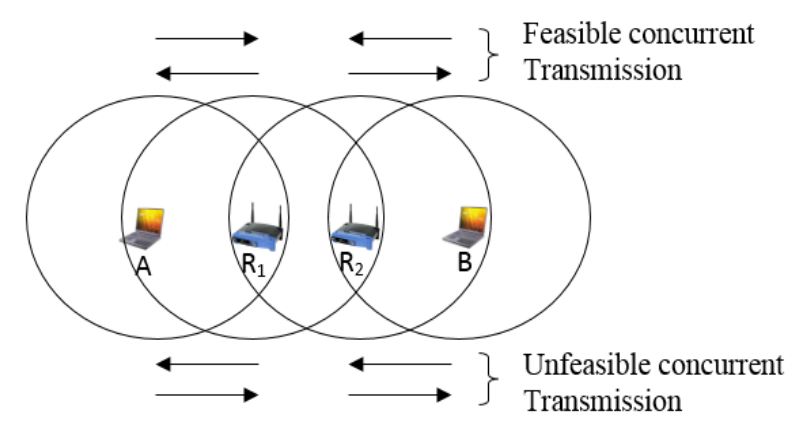

Fig 5: The Feasible and Unfeasible Concurrent Transmission Pattern 
However MACA-P is only performing well for specific scenario when large size of payload is considered. In addition, this protocol also caused wasted deferral time due to infeasible scheduling of concurrent transmission especially when low traffic is considered at intermediate nodes in multihop WMN communication.

\subsection{Concurrent Short Signaling Medium Access}

\section{Control (CSS-MAC) Protocol for Multihop}

\section{Wireless Mesh Network}

In this work [9, $10 \& 11]$, the author proposed an enhancements to the IEEE 802.11 MAC protocol to obtain higher concurrency that could reduce the amount of signaling overhead that required at each intermediate node until the frame forwarded to its end destination in multihop WMN. A MAC protocol namely Concurrent Short Signaling Medium Access Control (CSS-MAC) that allows the radio nodes to schedule concurrent transmission whenever possible (alleviate exposed node problem) and reduce the amount of signaling frames between intermediate nodes are designed and modeled analytically. The analytical modeling demonstrated the performance of the CSS-MAC protocol in term of delay and throughput when it is implemented in the multihop WMN scenario. The main aim of the proposed scheme is to provide better network performance and throughput. Since the function of a MAC protocol is to regulate and provide the equal right for all nodes in the network to access the channel, so the CSSMAC protocol was designed to cope this criterion. Figure 6 shows the pattern of the concurrent and short signaling transmission that could be allowed in CSS-MAC without any collision.

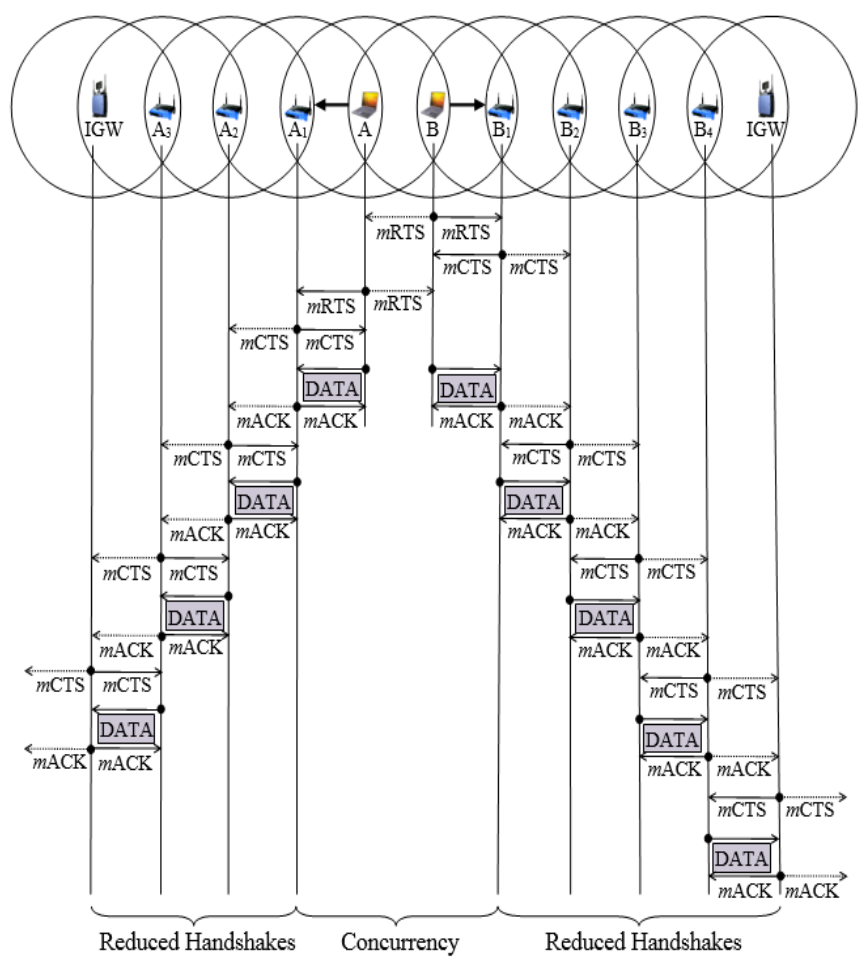

Fig 6: Basic Structure of the CSS-MAC Protocol

\section{FORWARDING ACCESS PROTOCOLS}

The background studies discovered the ineffectiveness of the existing IEEE 802.11 MAC in supporting the forwarding operation, especially when the multihop communication is concerned. Since this protocol is only designed to transmit or receive a frame in one hop manner, thus it performs poorly when directly implemented in multihop WMN communication. In addition, the use of RTS/CTS signaling frames at each hop caused large signaling overheads when the frame forwarded in multihop manner. This characteristic also degrades the throughput of the network significantly. An efficient forwarding access protocols have been proposed by many researchers to reduced signaling overhead caused by multihop environment. Three related approaches namely DCMA, Q-DCMA and MARCH that had been proposed to reduce overhead caused by signaling frames thus gain better throughput are discussed in the following section.

\subsection{Data-Driven Cut Through Medium Access} (DCMA)

To reduce the latency and improve the throughput in multihop networks, the Data-driven Cut through Medium Access protocol (DCMA) has been proposed [8]. The feature of this protocol is to reserve the channel for the next forwarding node by using new control frames called ACK/RTS as shown in Figure 7. This approach reduces the chance of frame collisions.

This protocol was designed with additional label in each frame to identify the next hop (i.e. downstream node) without negotiating with an IP lookup at routing layer. The protocol merges the ACK of ongoing transmission with the RTS control frame of next hop transmission (i.e. to the downstream node) which is the frame intended to. This new ACK/RTS now contain the MAC address of upstream node, downstream node and includes the information to find the next hop by the downstream node. Thus, in this way the inherent right to send an ACK frame now exploited by downstream node (i.e. next hop) to reserve the channel for its transmission.

According to this protocol, a network interface card (NIC) must quickly look the next hope address which is required to generate the ACK/RTS control frames. This operation must be done within the particular amount of time otherwise the communication would be discarded. The NIC architecture must be heavily modified in the DCMA protocol. In addition, it also entails additional information to be presented in NIC. Other than that, when the size of the network grows, the NIC memory also grows accordingly in this protocol. These are the drawbacks of the DCMA protocol. The other refinement of DCMA protocol can be found in [8]. 


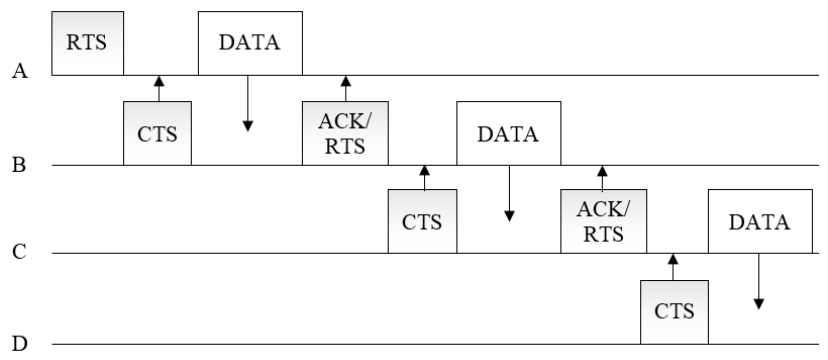

Fig 7: Data Driven Cut-Through MAC

\subsection{Queue - Data Driven Cut Through Medium}

\section{Access (Q-DCMA)}

Additionally, [13] showed the improvement of [8] by designing the cut-through ACK/RTS control frames. This protocol was designed with simpler hardware structure where it doesn't need the quick operation at the NIC as what DCMA needed. This protocol has the capability to picking the highest priority frame from the multiple queues. Thus this protocol can support certain degree of QoS. As both cut-through protocols can reduce the delay in multihop WMN, therefore network throughput can be improved accordingly.

\subsection{Medium Access With Reduce Handshake}

\section{(MARCH) Protocol}

Medium Access with Reduced Handshake (MARCH) protocol has been proposed to reduce the overhead of multihop network [14 \& 15] by using simpler reservation mechanism. MARCH is the new MAC protocol which is exploits the overhearing and broadcast characteristic of omni-directional antennas to reduce number of handshakes that require at each intermediate hops.

The RTS/CTS handshake only performed by the first hop of communicating nodes pair while the subsequent hops of communicating nodes pairs utilize a new signaling frame named as CTS-only. The RTS/CTS control frame is designed to hold additional information such as MAC address and Route ID ( $\mathrm{RT}_{\mathrm{ID}}$ ) in order to enable the forwarding operation. The subsequent intended hops will activate the timer based on this information. Once the timer is expired, the next hop subsequently invites its upstream node to relay the DATA frame via CTS-only frame. This scenario clearly elaborated by using Figure 8.

Since fewer signaling frames are exchanged over multiple hops, the overall throughput can be increased significantly. Moreover the probability of frame collision among the signaling frames has been reduced. However the protocol performs poorly when high traffic is considered in multihop network and when exposed node problem is occurred at the first hop of communicating nodes. The detail refinements of MARCH are described in [14 \& 15].

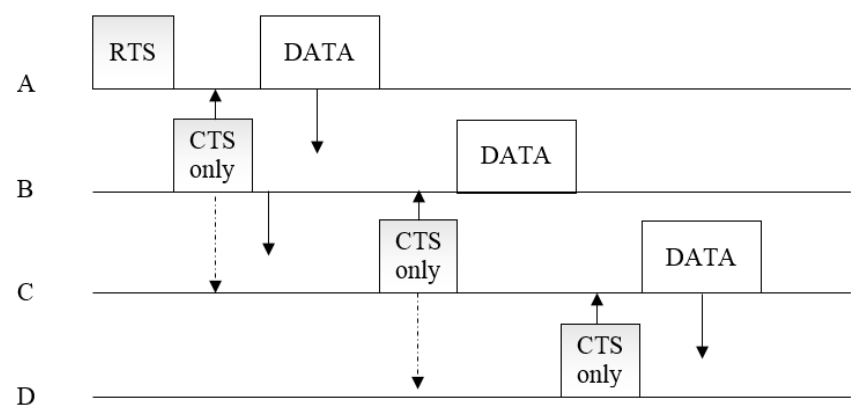

Fig 8: MARCH Access Protocol

\section{DISCUSSION}

By presenting inefficiencies (i.e. channel utilization and frame forwarding operation) that occurred in multihop WMN, it concluded that the existing 802.11 MAC protocol that is designed for single hop wireless LAN network does not function well when directly applied in the multihop WMN. In order to improve the efficiency of channel utilization, a concurrent transmission protocol known as MACA-P was proposed by Acharya in [5 \& 7]. The proposed technique mitigates the number of exposed node problem by allowing the concurrent transmission among the radio nodes in the network. However, it doesn't support the forwarding/relaying operation which is crucial in multihop WMN communication. The time consumed by MACA-P protocol at each intermediate relaying node to schedule the concurrent transmission increase the latency thus degrade the throughput significantly.

On the other hand, the techniques to reduce the control signaling overhead, the handshaking, were proposed in DCMA and MARCH protocol. These techniques are designed to receive the DATA frame and transmit it immediately to next hop without further control handshaking. The drawback of the DCMA protocol is requires a heavily modified NIC architecture and entails additional information to be presented in Network Interface Card (NIC). This caused the memory of NIC grows accordingly when the size of the network grows. In contrast, the MARCH protocol performs poorly when high traffic is considered in multihop network. This is due to exposed node problem which is occurred at each intermediate hop.

Meanwhile, CSS-MAC [9, 10, $11 \& 12]$ proposed a set of enhancement to the existing IEEE 802.11 MAC to solve both exposed node problem and signaling overhead issues by providing efficient channel utilization and forwarding operation. The significance of enabling the concurrent transmission and reducing the signaling overhead in different scenarios were presented in this work. The performance of the proposed CSS-MAC protocol compared and examined with other three different collision avoidance MAC protocols such as MACA-P, MARCH and IEEE 802.11 MAC. As far as end-to-end delay in multihop WMN is concerned, the existing MAC approaches seem to be inappropriate. The channel utilization and the forwarding operation with CSS-MAC protocol claimed to be more efficient, by enabling the concurrency and reducing the 
signaling overheads in multihop WMN environment. Thus, the CSS-MAC tackled both multiple access and multihop issues that degrade the performance in multihop WMN. More efficient MAC protocols to discover above issues has proposed in $[16,17,18,19 \& 20]$.

\section{CONCLUSION}

The background studies discovered the ineffectiveness of the existing IEEE 802.11 MAC protocol in solving exposed node issues and supporting the forwarding operation, especially when the multihop communication is concerned. An efficient MAC protocol is necessary to improve channel utilization in multihop WMN. As explained in this article, many innovative MAC protocols have been proposed by the researchers. Although many MAC protocols have been proposed, but desirable properties (i.e. delay and throughput) in either single hop or multihop networks it is not easy to satisfy. Therefore, the challenge in multihop WMN is to design a proper MAC protocol that could coordinate the channel access effectively to boost their performance.

\section{ACKNOWLEDGMENT}

The authors would like to take this opportunity to thanks those who are contributes directly or indirectly in completion of this article and also for their constructive comments. In addition, the authors also would like to express our gratitude to Centre for CoE-CeTRI: Telecommunication Research \& Innovation and also to Universiti Teknikal Malaysia Melaka (UTeM) for the support and encouragement.

\section{REFERENCES}

[1] IEEE, Institute of Electrical and Electronics Engineering "IEEE Standard for information Technology - Telecommunications and Information Exchange between Systems - Specific Requirements" - Part 5: Token Ring Access Method and Physical Layer Specifications. IEEE, New York, 1998.

[2] Tsai, T.J., \& Chen, J.W. "IEEE 802.11 MAC Protocol Over Wireless Mesh Networks : Problem and Perspective", Proceeding of the 19th International Conference on Advanced Information Networking and Application (AINA'05), 2005.

[3] Issariyakul, T., Hossain, E., \& Kim, DI, "Medium access control protocols for wireless mobile ad hoc networks: issues and approaches", Wireless Communications and Mobile Computing, pp. 935958, 2003.

[4] Tobagi, F., Kleinrock, L, "Packet Switching in Radio Channel: Part II - The Hidden Terminal Problem in Carrier Sense Multiple Access and the Busy - Tone Solution", IEEE Transactions on Communication, pp. 1417 - 1433.

[5] Acharya, A., Misra, A. \& Bansal, S, "MACA-P: A MAC for Concurrent Transmissions in Multi-hop Wireless Networks", IEEE International conference on Pervasive Computing and Communication (PerCom'03), 2003.
[6] Shukla, D., Chandran, W.L., \& Iyer, S., "Mitigating the exposed node problem" in IEEE 802.11 adhoc networks. IEEE ICCCN 2003, Dallas, 2003

[7] Acharya, A., Misra, A. \& Bansal, S., "Design and analysis of a cooperative medium access scheme for wireless mesh networks.” Proc. Intl. Conf. Broadband Netw. (BroadNets), San Jose, CA, 2004

[8] Acharya, A., Ganu, S., \& Misra, A., "DCMA: A Label Switching MAC for Efficient Packet Forwarding in Multihop Wireless Networks" in IEEE Journal on Selected Areas in Communications, pp. 1995 - 2004, 2006

[9] Vigneswara Rao, Gannapathy., Suaidi, Mohamad Kadim., Johal, Muhammad Syahrir Bin., Chuan, Lim Kim., Ramli, Nordin., Mohamad, Hafizal. "A Smooth Forwarding Operation in Wireless Mesh Network," in IEEE 10th Malaysia International Conference on Communications (MICC), pp. 83-87. 2011.

[10] Vigneswara Rao Gannapathy, Ahamed Fayeez Bin Tuani Ibrahim, Zahriladha Bin Zakaria, Abdul Rani Bin Othman, Mohamad Kadim Bin Suaidi, "Alleviate Exposed Node Issues In Wireless Mesh Network (WMN) Using A Novel Approach Of Concurrent Medium Access Control (C-MAC) Protocol", IJRET: International Journal of Research in Engineering and Technology eISSN: 2319-1163, pISSN: 2321-7308, Volume: 03 Issue: 11, Dec-2014

[11] Vigneswara Rao Gannapathy, Ahamed Fayeez Bin Tuani Ibrahim, Zahriladha Bin Zakaria, Abdul Rani Bin Othman, Anas Abdul Latiff, "An Enhancement Of RTS/CTS Control Handshake In CSMA/CA Based MAC Protocol For An Efficient Packet Delivery Over Multi-hop Wireless Mesh Network (WMN)", IJRET: International Journal of Research in Engineering and Technology eISSN: 2319-1163, pISSN: 2321-7308, Volume: 02 Issue: 10, Dec-2013.

[12] Vigneswara Rao Gannapathy, Tuani Ibrahim, Ahamed Fayeez, Zahriladha Zakaria, Abdul Rani Othman, Nur Qalbi Jalaudin, "A review on various types of Software Defined Radios (SDRs) in radio communication", IJRET: International Journal of Research in Engineering and Technology eISSN: 2319-1163, pISSN: 2321-7308, Volume: 03 Issue: 12, Dec-2014

[13] Raguin, D., Kubisch, M., Karl, H., \& Wolisz, A. "Queue - Driven Cut-Through Medium Access in Wireless Ad Hoc Networks" in IEEE Wireless Communication and Networking Conference (WCNC), 2004

[14] Toh, C.K., "Ad Hoc Mobile Wireless Networks: Protocol and System", 1st ed., NJ: Prentice Hall PTR, 2002.

[15] Toh, C.K., Vassiliou, V., Guichal, G., \& Shih, C.H., "MARCH: A Medium Access Control Protocol for Multihop Wireless Ad Hoc Networks", in Proceedings of IEEE Military Communication, pp. $512-516,2000$.

[16] F. V. Gallego, J. Alonso-Zarate, C. V. Verikoukis, and L. Alonso, "A survey on prototyping platforms for the development and experimental evaluation of 
medium access control protocols," IEEE Wireless Communications, vol. 19, no. 2, pp. 74-81, 2012.

[17] J. C. O’Sullivan, P. di Francesco, U. K. Anyanwu, L. A. SaSilva, and A. B. MacKenzie, "Multi-hop MAC Implementations for Affordable SDR Hardware," in Proceedings of the IEEE Symposium on New Frontiers in Dynamic Spectrum Access Newtorks, Aachen, Germany, 2011.

[18] J. Ansari, X. Zhang, S. Gaikwad, and P. Mah“ onen, "Exploring MAC Parallelization on Software Defined Radio Platforms," in Proceedings of the IEEE International Conference on Sensing, Communication, and Networking, New Orleans, USA, 2013.

[19] A.T.I Fayeez, V.R Gannapathy, S. S. S Ranjit, S.K. Subramaniam, Ida S.Md Isa, "Throughput Analysis Of Energy Aware Routing Protocol For Real-Time Load Distribution In Wireless Sensor Network (WSN)", IJRET: International Journal of Research in Engineering and Technology eISSN: 2319-1163, pISSN: 2321-7308, Volume: 02 Issue: 11, Nov-2013.

[20] Abdulaziz M. Ghaleb, David Chieng, Alvin Ting, Seh Chun Ng, Ayad Abdulkafi, Kim-Chuan Lim, HengSiong Lim, "Preservation of QoS Across Hybris LTE-WLAN Routers", IET International Conference on Information and Communications Technologies (IETICT), Beijing, April 2013.

\section{BIOGRAPHIES}

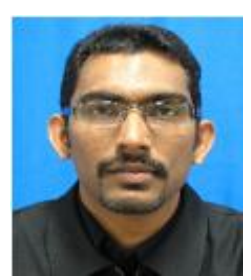

Vigneswara Rao Gannapathy currently serving as a lecturer in Universiti Teknikal Malaysia Melaka (UTeM) and he actively involves in research activities which is related to Electronics and Wireless Communication. His research interest and direction has focused on $5 \mathrm{G}$ Wireless Communication Systems, IoT and Big Data that emerged as a key technology for next - generation Wireless Communication.

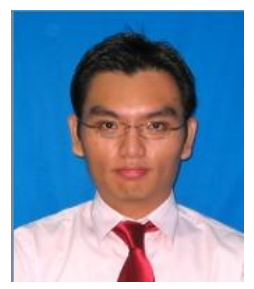

Lim received his B.Eng (2003), MSc. (2005), and PhD. (2009) degrees from the Faculty of Arts, Computing, Engineering and Sciences, Sheffield Hallam University., UK. From 2005 he was a research assistant in the Mobile Machines and Vision Laboratory (MMVL) at Sheffield Hallam University. He is currently a senior lecturer at the FKEKK, UTeM. Malaysia

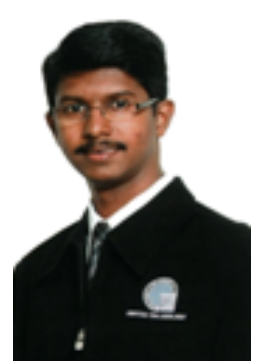

Siva Kumar Subramaniam graduated with B.Eng (Hons) and M.Sc in Electronics Engineering from Universiti Teknikal Malaysia, Melaka in 2006 and 2009, respectively.His research interest is in wireless sensor network for long range data transmission using IEEE 802.11 and IEEE 802.15.4 standards.

Dr. Zahriladha Zakaria, PhD, MIEEE,

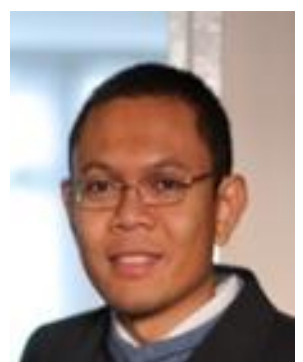

BEM, Grad IEM is currently working as a lecturer at the Faculty of Electronic and Computer Engineering, University Teknikal Malaysia Melaka. (UTeM). His research interests include a variety of microwave device development such as planar and non-planar microwave filters, amplifiers and antennas. 